\title{
Feasibility of microbial sample collection on the skin from people in Yaoundé, Cameroon
}

\author{
Nana C. Benderli ${ }^{1,2,}$, Kazuhiro Ogai ${ }^{3, \S}$, Yukie M. Lloyd ${ }^{4}$, John Paul Arios ${ }^{4}$, Boonyanudh Jiyarom ${ }^{4}$, \\ A. Honore Awanakam ${ }^{1}$, Livo Forgu Esemu ${ }^{1}$, Aki Hori ${ }^{5}$, Rosette Megnekou ${ }^{1,2}$, Rose G.F. Leke ${ }^{1}$, \\ Takayuki Kuraishi, ${ }^{5, *}$, Shigefumi Okamoto ${ }^{3, *}$, Gabriel Loni Ekali ${ }^{1,6}$ \\ ${ }^{1}$ The Biotechnology Center, University of Yaoundé I, Yaoundé, Cameroon; \\ ${ }^{2}$ Department of Animals Biology and Physiology, University of Yaoundé I, Yaoundé, Cameroon; \\ ${ }^{3}$ Faculty of Health Sciences, Institute of Medical, Pharmaceutical and Health Sciences, Kanazawa University, Ishikawa, Japan; \\ ${ }^{4}$ Department of Tropical Medicine, Medical Microbiology and Pharmacology, John A. Burns School of Medicine, University of \\ Hawaii at Manoa, Honolulu, Hawaii, U.S.A.; \\ ${ }^{5}$ Department of Faculty of Pharmacy, Institute of Medical, Pharmaceutical and Health Sciences, Kanazawa University, Ishikawa, \\ Japan; \\ ${ }^{6}$ National AIDS Control Committee, Yaoundé, Cameroon.
}

Summary Characterization of microbial communities in the skin in healthy individuals and diseased patients holds valuable information for understanding pathogenesis of skin diseases and as a source for developing novel therapies. Notably, resources regarding skin microbiome are limited in developing countries where skin disorders from infectious diseases are extremely common. A simple method for sample collection and processing for skin microbiome studies in such countries is crucial. The aim of this study is to confirm the feasibility of collecting skin microbiota from individuals in Yaoundé, a capital city of Cameroon, and subsequent extraction of bacterial DNA in a resource limited setting. Skin swabs from several individuals in Yaoundé were successfully obtained, and sufficient amount of bacterial 16S ribosomal RNA-coding DNA was collected, which was confirmed by quantitative PCR. The median copy number of $16 \mathrm{~S}$ ribosomal RNA gene varied across participants and collection sites, with significantly more copies in samples collected from the forehead compared to the left and right forearm, or back. This study demonstrated that collecting surface skin microbes using our swabbing method is feasible in a developing country. We further showed that even with limited resources, we could collect sufficient amount of skin microbiota from the inhabitants in Yaoundé where no studies of skin microbiome were reported, which can be passed to further metagenomic analysis such as next generation sequencing.

Keywords: Skin swabs, surface skin microbiome, developing countries, Cameroon

${ }^{\S}$ These authors contributed equally to this work.

*Address correspondence to:

Dr. Takayuki Kuraishi, Faculty of Pharmacy, Institute of Medical, Pharmaceutical and Health Sciences, Kanazawa University, Kakuma-machi, Kanazawa, Ishikawa 920-1192, Japan.

E-mail: tkuraishi@staff.kanazawa-u.ac.jp

Dr. Shigefumi Okamoto, Faculty of Health Sciences, Institute of Medical, Pharmaceutical and Health Sciences, Kanazawa University, 5-11-80 Kodatsuni, Kanazawa, Ishikawa 9200942 , Japan.

E-mail: sokamoto@mhs.mp.kanazawa-u.ac.jp

\section{Introduction}

The skin is one of the largest organ of human beings. In addition to its role in physical protection and temperature regulation, it serves as a unique and dynamic ecosystem that is inhabited by over $10^{10}$ microorganisms (i.e., skin microbiota) (1). In a homeostatic environment, the skin microbiota are harmless and play a protective and/or regulatory role by controlling the innate and adaptive immune systems found in our skin (2). However, when the 
environment on the skin is altered, the composition of skin microbiota can also be disrupted: a phenomenon known as dysbiosis. Studying the type of dysbiosis, for example, whether the alteration occurs at the species or strain level, provides important information for understanding skin pathology, developing a diagnostic tool and an appropriate treatment (3).

A number of studies on the association between dysbiosis and skin disorders such as atopic dermatitis (4), psoriasis (5), seborrheic dermatitis (6) and acne vulgaris (7) have been published. However, how dysbiosis of the skin microbiome relates to tropical diseases that affect skin integrity is understudied. For example, there are only a few studies to date that report dysbiosis in the skin of patients with leprosy $(8,9)$ or leishmaniasis $(10)$. Whether other skin-affecting tropical diseases or neglected infectious diseases (e.g., tuberculosis, filariasis, schistosomiasis), as well as the infection of human immunodeficiency virus (HIV) and acquired immunodeficiency syndrome (AIDS) that may alter the skin immune system, could interact with the dysbiosis of the skin microbiota remains unclear.

The major limitation for studying the skin microbiome is the complexity of sample collection, such as consistency in collection methods and contamination risks (11), handling and storage of samples. Processing of microbial DNA is also a challenge $(12,13)$, due to the relatively low abundance of skin microbiome compared to the gut. Along with these complexities, many developing countries in Africa also face issues with limited resources and inadequate research facilities to carry out such studies (14). Thus, it is desirable to establish a simple protocol for collecting skin samples that is feasible to carry out in a basic laboratory in a resource-limited country without compromising the quantity and quality of the microbial DNA collected. The development of such a protocol will lead to ensuring the consistency of techniques employed in the field and guarantee of the quality of data acquired.

To achieve this objective, this study attempted to evaluate the feasibility of collecting and processing skin samples at a hospital and university research laboratory in Yaoundé, Cameroon with basic equipment. A noninvasive sample collection method was employed by utilizing flocked swabs (15) to confirm the presence of enough amount of bacterial DNA coding for $16 \mathrm{~S}$ ribosomal RNA (rRNA).

\section{Materials and Methods}

\subsection{Ethical consideration}

This study was approved by the National Ethics Committee of Cameroon, (Ethical Clearance 2018/06/ $\mathrm{N}^{\circ} 1045 / \mathrm{CE} / \mathrm{CNERSH} / \mathrm{SP}$ ) and an authorization was obtained from the hospital administration before participants were recruited. Participation in the study was voluntary and written informed consent was obtained from each participant prior to enrollment. Oral consent and signature from a guardian were provided for those who were physically unable to provide written consent. The study was performed in strict respect of the guidelines for human clinical research and all data were decoded for anonymity.

\subsection{Collection site and sample population}

This study was part of a larger cross-sectional study that took place from June to October 2018 at the HIV clinic of the Efoulan District Hospital in Yaoundé, Cameroon. The Efoulan District Hospital was chosen due to an existing collaboration with the hospital and university research center. In the original study, out-patients, inpatients and hospital staff were enrolled for sampling. HIV-uninfected, healthy subjects were defined by the absence of any known skin pathology and a negative HIV rapid diagnostic test (RDT) result; HIV-infected subjects were defined by a positive HIV RDT result. Pregnant women and those who had apparent skin disorders that are not associated with HIV or AIDS, such as atopic dermatitis and psoriasis, were excluded.

\subsection{Sample collection}

Skin swabs were collected using the Puritan HydraFlock Sterile Flocked Swabs (25-3306-H, Puritan Medical Products Company, ME, USA). Prior to swabbing, 1.5$\mathrm{mL}$ microcentrifuge tubes were filled with $100 \mu \mathrm{L}$ of sterile $0.9 \%$ sodium chloride solution (S5815; Teknova, CA, USA) with $0.1 \%$ Tween 20 (28353-14; Nacalai Tesque, Inc., Kyoto, Japan). First the swab head was soaked in the solution in the tube $(16,17)$. Swabbing was then performed in a $5 \times 5 \mathrm{~cm}$-square on designated sites (Figure S1, http://www.ddtjournal.com/action/ getSupplementalData.php?ID=51). The patients with Kaposi's sarcoma were additionally swabbed at lesion sites. After swabbing, each swab head was placed into the original tubes (Figure S2, http://www.ddtjournal. com/action/getSupplementalData.php? ID=51). Samples were stored in a cooler box and transferred to the laboratory for DNA extraction.

\subsection{DNA extraction from skin swab samples}

DNA was extracted from samples using the Kaneka Easy DNA Extraction Kit version 2 (KN-T110005; Kaneka Corp., Tokyo, Japan), which contains DNA extraction Solutions A and B, according to the manufacturer's instructions (Figure S2, http:// www.ddtjournal.com/action/getSupplementalData. php?ID=51). Briefly, $200 \mu \mathrm{L}$ of Solution A was added into each microcentrifuge tube containing the swab head and was mixed by vortexing. Next, sample tubes were placed in a water bath and incubated for $9 \mathrm{~min}$ at 
$98^{\circ} \mathrm{C}$. The samples were cooled to room temperature, and $28 \mu \mathrm{L}$ of Solution B was added to each tube. After mixing briefly by vortexing, samples were stored at $-20^{\circ} \mathrm{C}$ until further analysis.

\subsection{Qualitative PCR amplification of $16 S$ rRNA gene}

To confirm whether bacterial DNA was successfully obtained, qualitative PCR amplification of the $16 \mathrm{~S}$ rRNA gene was performed using SapphireAmp Fast PCR Master Mix (RR350A, Takara Bio Inc., Shiga, Japan) and primers (forward: 341F, 5'-CCTACGGGNGGCWGCAG-3'; reverse: 806Rmod, 5'-GACTACHVGGGTATCTAAKCC-3'; targeting $460-$ bp V3-V4 region of the $16 \mathrm{~S}$ rRNA gene). One microliter of the template DNA was used in the 10 $\mu \mathrm{L}$ reaction mixture. The PCR protocol was set as: $94^{\circ} \mathrm{C}$ for $1 \mathrm{~min} ; 33$ cycles of $98^{\circ} \mathrm{C}$ for $10 \mathrm{sec}, 55^{\circ} \mathrm{C}$ for $10 \mathrm{sec}$, and $72^{\circ} \mathrm{C}$ for $15 \mathrm{sec}$; and $72{ }^{\circ} \mathrm{C}$ for 1 min. The PCR products were then electrophoresed by using i-MyRun II (IMR2-001, CosmoBio Co., Ltd., Tokyo, Japan) with 2\% Agarose S (312-01193, FUJIFILM Wako Pure Chemical Corp., Osaka, Japan) gel, stained by $0.5 \mu \mathrm{g} / \mathrm{mL}$ ethidium bromide (31590051, FUJIFILM Wako Pure Chemical Corp.), and visualized using LuminoGraph I (WES-6100, ATTO Corp., Tokyo, Japan). The presence of a band at the expected size ( $460 \mathrm{bp})$ for each sample was determined positive.

\subsection{DNA clean up}

To exclude the possibility of PCR inhibition, DNA samples were cleaned using a NucleoSpin gDNA Clean-up kit (740230, Macherey-Nagel GmbH \& Co. $\mathrm{KG}$, Düren, Germany) according to the manufacturer's instructions. The samples were stored at $-20^{\circ} \mathrm{C}$ until further analysis.

\subsection{Quantitative PCR}

Quantitative real-time PCR (qPCR) was performed as described elsewhere (18). In brief, the DNA samples were amplified with $16 \mathrm{~S}$ universal primer pairs (F: 5'-ACTGAGAYACGGYCCA-3'; R: 5'-CTGCTGGCACGDAGTTAGCC-3') and a $16 \mathrm{~S}$ universal probe (5'-VIC-ACTGCTGCCTCCCGTANFQ-MGB-3') by using the Thunderbird Probe qPCR Mix (Toyobo Co., Ltd., Osaka, Japan). A standard curve was drawn from a known amount of the $16 \mathrm{~S}$ rRNA gene. All the reactions were performed with the LightCycler 480 System (F. Hoffmann-La Roche, Ltd., Basel, Switzerland).

\subsection{Statistical data analysis}

Data are shown as the 25 th, 50 th, 75 th percentile boxes with 25 th percentile $-1.5 \times$ interquartile range $(\mathrm{IQR})$ to 75 th percentile $+1.5 \times$ IQR whisker in the box plot. The box plot with dots was created using the $\mathrm{R}$ statistical software (version 3.6.0). The differences in the copy number of the $16 \mathrm{~S}$ rRNA gene among groups were compared by using the Kruskal-Wallis test followed by the Steel-Dwass post hoc multiple comparison test using R. A $p$-value $<0.05$ was considered statistically significant.

\section{Results and Discussion}

In Yaoundé, Cameroon, we successfully obtained skin swab samples of head, left and right arms and back from $26 \mathrm{HIV}$-negative healthy individuals (males: 7, females: 19; age 21-51, median 27) and 25 HIV-positive patients (males: 5, females: 20; age 22-61, median 39). Of all the HIV-positive patients, two patients presented with Kaposi's sarcoma, and swabs from the sarcoma lesions were also obtained from those patients.

There are multiple skin microbiome sampling methods, including swabs, biopsies, surface scrapes, cup scrubs and tape strips $(18,19)$. Each method varies in biomass yield, human DNA contribution, sampling depth and discomfort level. Since patients with skin disorder are commonly sensitive to skin stimulation, it is difficult to tolerate even minimal pain that accompanies invasive methods. To overcome this problem, using pre-moistened skin swabs was our method of choice. As a result, no skin-related troubles were reported when collecting skin samples, even in patients who were immunosuppressed with later stages of AIDS.

Next, we attempted to confirm if the extracted DNA can be used for subsequent bacterial DNA analysis (e.g., next generation sequencing [NGS]) by qualitative PCR for the bacterial $16 \mathrm{~S}$ rRNA gene. Although crudely extracted DNA samples processed just after the DNA extraction still contain debris of skin cells, sebum, and dirt, we found that nine out of 18 representative samples were successfully amplified by PCR (Figure S3, http:// www.ddtjournal.com/action/getSupplementalData. $p h p ? I D=51)$. Lack of amplicons is likely due to the presence of skin cells, sebum or other debris, which could interfere with the PCR amplification process of extracted DNA. Contamination of these objects can be explained by the lack of washing steps for the kit used in this study; which is otherwise beneficial by reducing the burden of sample processing.

To minimize any interference from heavy contamination of human cell debris and/or sebum, all crude DNA samples were cleaned with a gDNA cleaning kit. After cleaning of crude DNA samples, the targeted area of 16S rRNA gene was successfully amplified for all samples tested without any nonspecific products, regardless of swabbing area (Figures 1 and S3, http://www.ddtjournal.com/action/ 
A

A $\quad \frac{\mathrm{HIV}^{+}-1}{\mathrm{~A} \text { B C C D A B C D A B C D }} \frac{\mathrm{HIV}^{+}-2}{\text { A B C C D KS A B C D KS NTC }}$

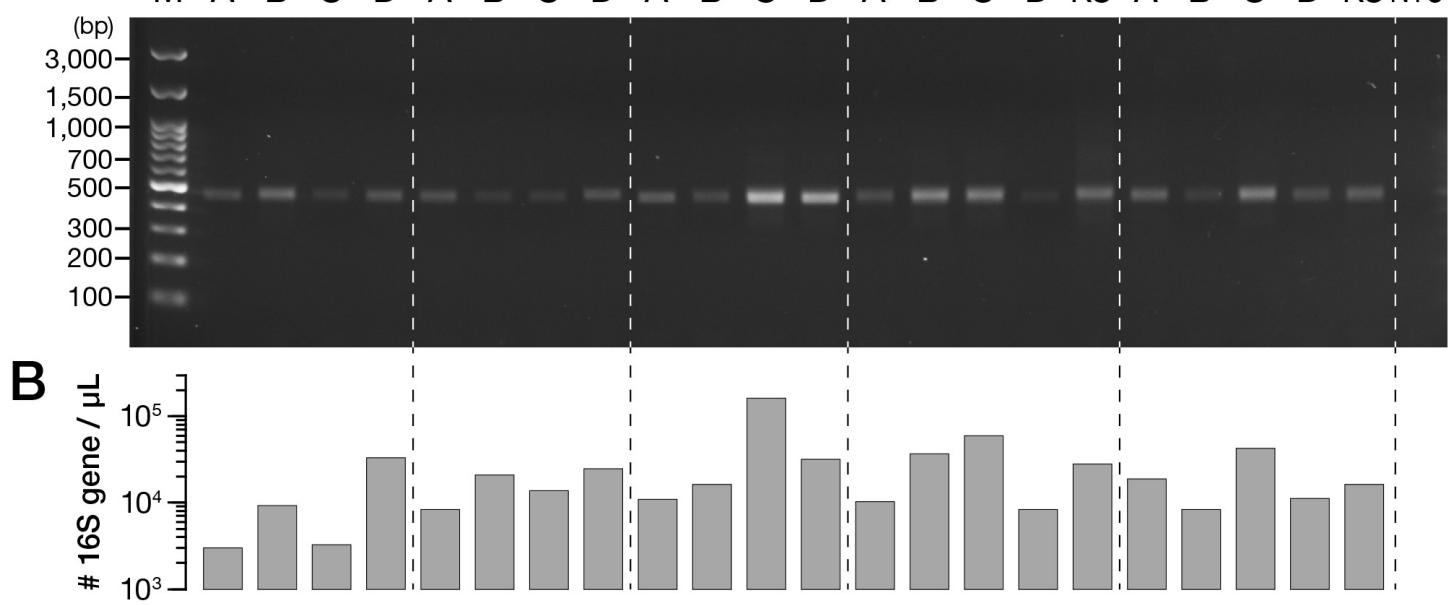

Figure 1. Successful amplification of bacterial 16S rRNA gene from skin swabs obtained from Cameroonians at the Efoulan district hospital. (A) Representative amplicons of the 16S rRNA gene (covering V3-V4 region, expected size $\sim 460$ bp) derived from two HIV-positive patients, one healthy individual, and two AIDS patients were electrophoresed and visualized. (B) The numbers of 16S rRNA gene in each sample. Each individual was sampled from four regions of interest, namely, the forehead (denoted as A), right forearm (B), left forearm (C), and mid-upper back (D). Skin swabs on the lesion of Kaposi's sarcoma in AIDS patients were also analyzed and visualized (KS). M: 100 bp marker, NTC: no template control.

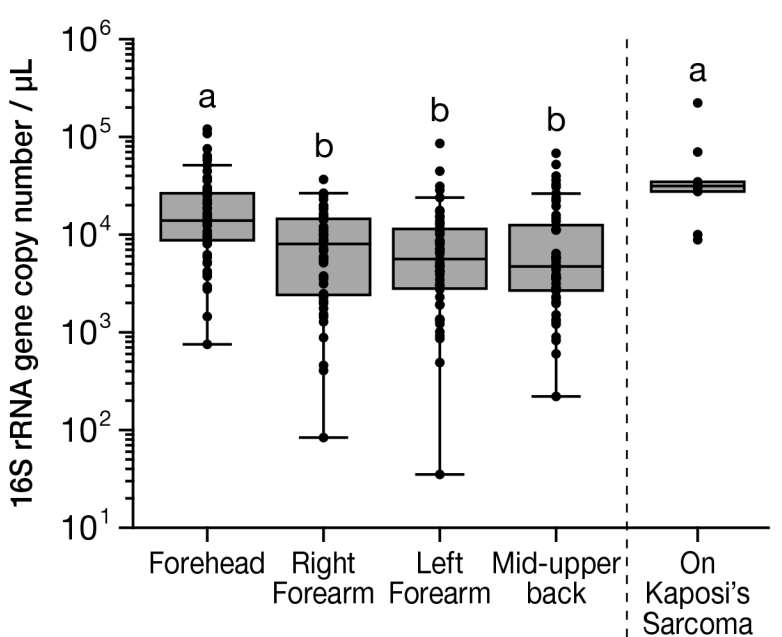

Figure 2. Copy number of obtained bacterial 16S rRNA gene. Although the amount of 16S rRNA gene varied among participants and collection sites, sufficient amount of $16 \mathrm{~S}$ rRNA gene was successfully obtained. The same letters indicate no significant difference, whereas different letters indicate significant difference $(p<0.05)$ between the groups.

getSupplementalData.php?ID=51). Bands for two samples, 10D and 40B, were extremely faint but present as confirmed by qPCR. For samples $19 \mathrm{C}$ and 40B, the signals diminished after cleaning, probably due to technical error.

We next quantified the copy number of bacterial 16S rRNA gene in each sample. Copy numbers of $16 \mathrm{~S}$ rRNA gene slightly differed depending on the individual and body parts of collection; samples collected from the forehead and the lesion of Kaposi's sarcoma showed significantly higher copy numbers of the $16 \mathrm{~S}$ rRNA gene (Figure 2 and Table 1). Except for a few samples ( 2 samples showed $<100$ copies $/ \mu \mathrm{L}$ ), we confirmed
Table 1. $p$-values of the multiple comparison in the number of $16 \mathrm{~S}$ copy number

\begin{tabular}{lcccc}
\hline & Forehead & $\begin{array}{c}\text { Right } \\
\text { forearm }\end{array}$ & $\begin{array}{c}\text { Left } \\
\text { forearm }\end{array}$ & $\begin{array}{c}\text { Mid-upper } \\
\text { back }\end{array}$ \\
\hline Right forearm & 0.003 & - & - & - \\
Left forearm & $<0.001$ & 0.94 & - & - \\
Mid-upper back & $<0.001$ & 0.95 & 1.00 & - \\
Kaposi's sarcoma & 0.22 & 0.002 & 0.001 & 0.004 \\
\hline
\end{tabular}

Please refer to Figure 2.

that the amounts of the 16S rRNA gene were sufficient enough for further analysis. The median copy number of bacterial 16S rRNA gene varied across participants and collection sites, with significantly more copies in samples collected from the forehead compared to the right forearm $(p=0.003)$, left forearm $(p<0.001)$ or back $(p<0.001)$ (Figure 2 and Table 1$)$. This variation could be explained by the frequency of exposure to the external environment (especially for the forehead) or physiological differences across sampled skin types: such as moisture or oiliness, that could harbor groups or quantity of distinct microbes (20).

The skin microbiota can provide colonization resistance, hindering pathogenic microbes from colonizing the skin, by competing for space or metabolites and by creating a hostile environment $(1,20,21)$. Dysbiosis in the skin could disrupt colonization resistance and introduce a niche for pathogenic microbes to thrive. Whether the dysbiosis in skin microbiota leads to worsened skin disorders or vice versa is unknown; and a longitudinal study would be necessary to provide an answer. A simple method for sample collection and processing is crucial to conduct longitudinal studies in resource-limited countries; and our study showed that sufficient quantity 
and quality of bacterial 16S rRNA gene for downstream applications can be collected using a non-invasive swab method.

We conclude that skin sampling with pre-moistened cotton is the valid method for collecting skin surface bacterial samples both in healthy people and those with skin disorders in resource-limiting situation such as in developing countries.

\section{Acknowledgements}

We thank the staff of the Efoulan district hospital for their collaboration and support, especially Dr Akame, the HIV clinic coordinator. We also thank the researchers and laboratory staff at the Biotechnology Centre, University of Yaoundé I, Cameroon, for processing and archiving the samples, and Ms. Ayaka Matsuoka for archiving samples, creating sample inventory, running gel electrophoresis and qPCR. Thanks to Dr. Ivo Sah Bandar and Eva ML for proofreading the manuscript. We appreciate all the participants of this study for their time and contribution. This research was supported by grants: International Research Training Program, National Institute on Minority Health and Health Disparities (T37MD008636); Global Infectious Disease Research Training, Fogarty International Center, National Institutes of Health (NIH) (D43TW009074); Centers of Biomedical Research Excellence, National Institute of General Medical Sciences, NIH (P30GM114737); National Institute of Allergy and Infectious Diseases, NIH (1R21 AI105286); Japan Society for the Promotion of Science Kakenhi (17H04428); and Kanazawa University Sakigake Project. YML's travels were supported by the Career Development Award from the American Association of University Women Hawaii Branch. KO's travel and accommodation expenses were supported in part by the Overseas Research Support for Young Scientists in Kanazawa University.

\section{References}

1. Belkaid Y, Segre JA. Dialogue between skin microbiota and immunity. Science. 2014; 346:954-959.

2. Belkaid Y, Tamoutounour S. The influence of skin microorganisms on cutaneous immunity. Nat Rev Immunol. 2016; 16:353-366.

3. Grice EA. The skin microbiome: potential for novel diagnostic and therapeutic approaches to cutaneous disease. Semin Cutan Med Surg. 2014; 33:98-103.

4. Bjerre RD, Bandier J, Skov L, Engstrand L, Johansen JD. The role of the skin microbiome in atopic dermatitis: a systematic review. Br J Dermatol. 2017; 177:1272-1278.

5. Langan EA, Griffiths CEM, Solbach W, Knobloch JK, Zillikens D, Thaci D. The role of the microbiome in psoriasis: moving from disease description to treatment selection? Br J Dermatol. 2018; 178:1020-1027.

6. Tanaka A, Cho O, Saito C, Saito M, Tsuboi R, Sugita T. Comprehensive pyrosequencing analysis of the bacterial microbiota of the skin of patients with seborrheic dermatitis. Microbiol Immunol. 2016; 60:521-526.

7. Fitz-Gibbon S, Tomida S, Chiu BH, et al. Propionibacterium acnes strain populations in the human skin microbiome associated with acne. J Invest Dermatol. 2013; 133:2152-2160.

8. Silva PE, Costa PS, Avila MP, Suhadolnik ML, Reis MP, Salgado AP, Lima MF, Chartone-Souza E, Nascimento AM. Leprous lesion presents enrichment of opportunistic pathogenic bacteria. Springerplus. 2015; 4:187.

9. Silva PES, Reis MP, Avila MP, Dias MF, Costa PS, Suhadolnik MLS, Kunzmann BG, Carmo AO, Kalapotakis E, Chartone-Souza E, Nascimento AMA. Insights into the skin microbiome dynamics of leprosy patients during multi-drug therapy and in healthy individuals from Brazil. Sci Rep. 2018; 8:8783.

10. Gimblet C, Meisel JS, Loesche MA, Cole SD, Horwinski J, Novais FO, Misic AM, Bradley CW, Beiting DP, Rankin SC, Carvalho LP, Carvalho EM, Scott P, Grice EA. Cutaneous leishmaniasis induces a transmissible dysbiotic skin microbiota that promotes skin inflammation. Cell Host Microbe. 2017; 22:13-24.e14.

11. Salter SJ, Cox MJ, Turek EM, Calus ST, Cookson WO, Moffatt MF, Turner P, Parkhill J, Loman NJ, Walker AW. Reagent and laboratory contamination can critically impact sequence-based microbiome analyses. BMC Biol. 2014; $12: 87$.

12. Human Microbiome Project Consortium. A framework for human microbiome research. Nature. 2012; 486:215221.

13. Kong HH, Andersson B, Clavel T, Common JE, Jackson SA, Olson ND, Segre JA, Traidl-Hoffmann C. Performing skin microbiome research: a method to the madness. J Invest Dermatol. 2017; 137:561-568.

14. Ngwarai MR, Ah Tow LE, Nicol MP, Kaba M. The human microbiome research in Africa - A systematic review. Int J Infect Dis. 2016; 45:143.

15. Warnke P, Warning L, Podbielski A. Some are more equal--a comparative study on swab uptake and release of bacterial suspensions. PLoS One. 2014; 9:e102215.

16. Grice EA, Kong HH, Conlan S, Deming CB, Davis J, Young AC, Program NCS, Bouffard GG, Blakesley RW, Murray PR, Green ED, Turner ML, Segre JA. Topographical and temporal diversity of the human skin microbiome. Science. 2009; 324:1190-1192.

17. Capone KA, Dowd SE, Stamatas GN, Nikolovski J. Diversity of the human skin microbiome early in life. J Invest Dermatol. 2011; 131:2026-2032.

18. Ogai K, Nagase S, Mukai K, Iuchi T, Mori Y, Matsue M, Sugitani K, Sugama J, Okamoto S. A comparison of techniques for collecting skin microbiome samples: swabbing versus tape-stripping. Front Microbiol. 2018; 9:2362.

19. Grice EA, Kong HH, Renaud G, Young AC, Program NCS, Bouffard GG, Blakesley RW, Wolfsberg TG, Turner ML, Segre JA. A diversity profile of the human skin microbiota. Genome Res. 2008; 18:1043-1050.

20. Grice EA, Segre JA. The skin microbiome. Nat Rev Microbiol. 2011; 9:244-253.

21. Sanford JA, Gallo RL. Functions of the skin microbiota in health and disease. Semin Immunol. 2013; 25:370-377.

(Received October 4, 2019; Revised December 19, 2019; Accepted December 21, 2019) 